\title{
Research Paper \\ Effect of Progressive Muscle Relaxation on Social Performance and Quality of Life in Aging
}

\author{
Ali Hasanpour-Dehkordi ${ }^{1}, *$ Ali Akbar Rastar ${ }^{2}$
}

1. Department of Medical Surgical Nursing, Faculty of Nursing and Midwifery, Shahrekord University of Medical Sciences, Shahrekord, Iran.

2. Department of Nursing, Faculty of Nursing and Midwifery, Shahrekord University of Medical Sciences, Shahrekord, Iran.

Crtation: Hasanpour-Dehkordi A, Rastar AA. [Effect of progressive muscle relaxation on social performance and quality of life in ageing (Persian)]. Iranian Journal of Ageing. 2016; 11(2):244-249. http://dx.crossref.org/10.21859/sija-1102244

doi : http://dx.crossref.org/10.21859/sija-1102244

Received: 18 Apr. 2016 Accepted: 10 Jun. 2016

Key words:

Performance, Relaxation, Quality of life, Ageing

\section{A B STRACT}

Objectives Aging is an important stage of life that is influenced by several factors such as mobility and exercise as they can promote healthy aging. The present study aimed to examine the effects of progressive muscle relaxation on the quality of life of elderly people living in Shahr-e-Kord, Iran.

Methods \& Materials In this quasi-experimental study, the sample was randomly divided into two groups: test and control. Participants in the test group were instructed to systematically tense muscle groups in a specific part of their body. They were then required to take a slow and deep breath and squeeze the muscles as hard as possible for five seconds followed by quickly relaxing the tensed muscles. Each session lasted for approximately 45 minutes. The intervention was implemented for three days in a week spanning across three months.

Results The results showed progressive muscle relaxation and enhanced quality of life in the intervention group. In addition, statistically significant differences were noted in the mean scores of physical performance, restricted activity after physical problem, energy, socially function, physical pain, overall hygiene, and quality of life between the intervention and control groups.

Conclusion The findings of this study revealed that the progressive muscle relaxation technique can effectively reduce the duration of rehabilitation, days of hospitalization and healthcare costs, as well as improve the quality of life, mood, and mental health in the elderly people. 


\title{
بررسى تأثير آرامسازى ييش رونده عضلانى بر عملكرد اجتماعى و ابعاد مختلف كيفيت زندتى سالمندان
}

\author{
على حسنيور دهكردى'، "على اكبر رستار”

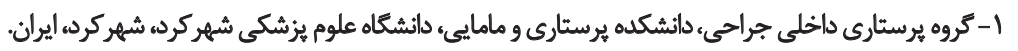

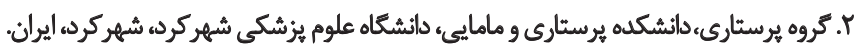

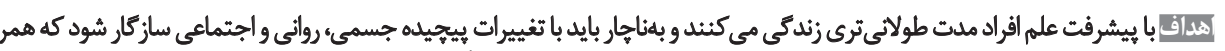

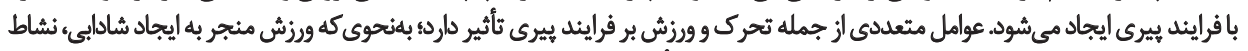

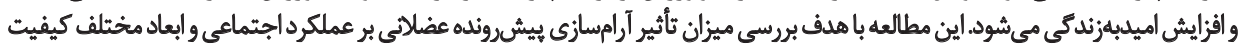

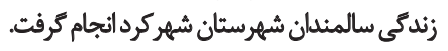

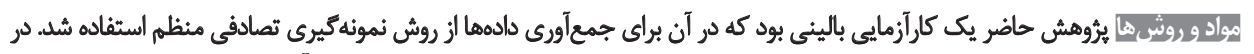

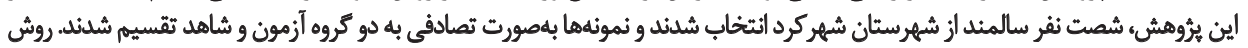

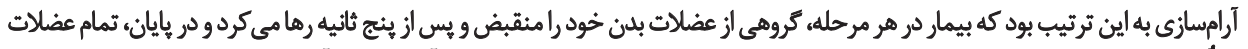

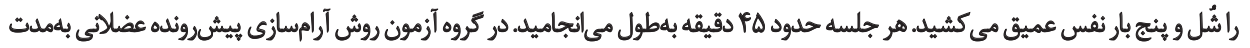

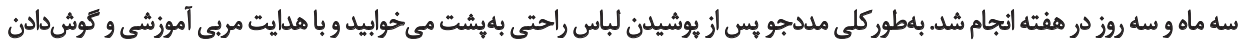

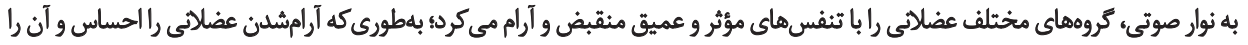

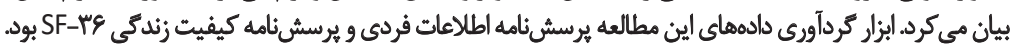

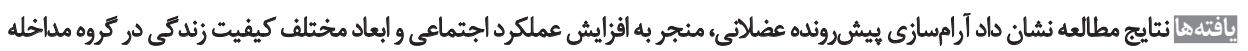

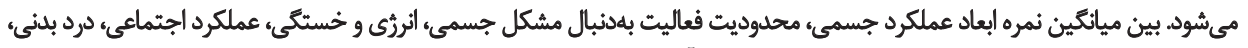

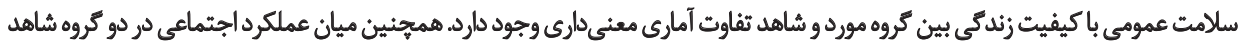

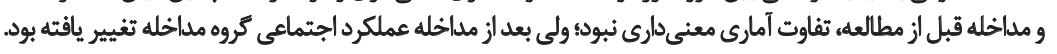

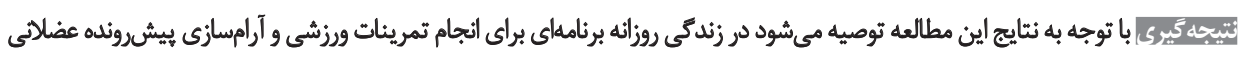

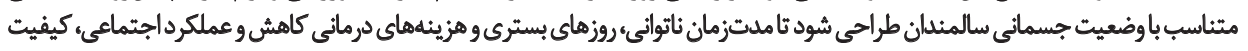

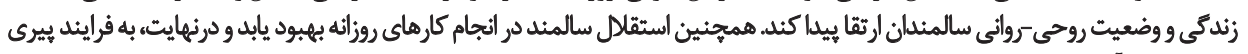

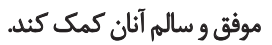

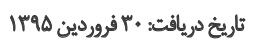

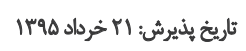

كليدوأوها:

آرامسازى ييشرونده عضلاتى، كيفيت زندكي، سالمند

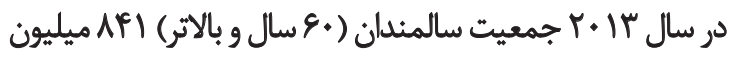

مقدمه

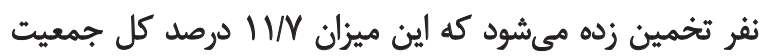

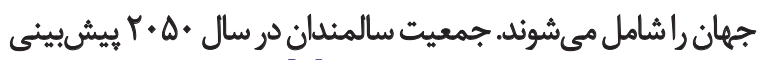

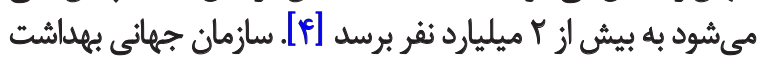

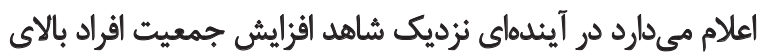

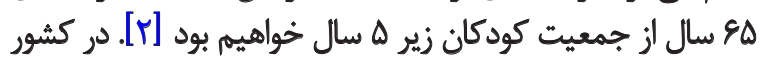

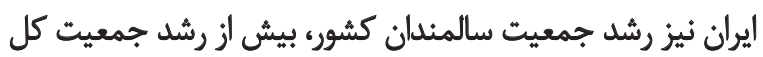

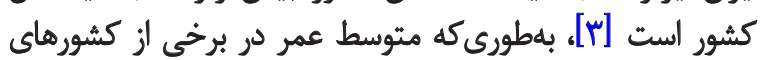

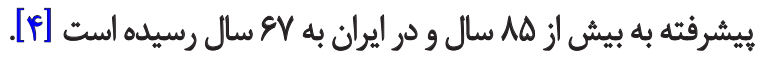

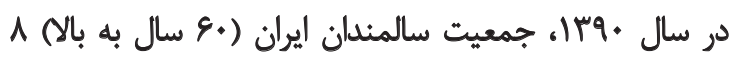
دوران سالمندى موفق و بانشاط، مستلزم رفاه كامل در همه ابعاد

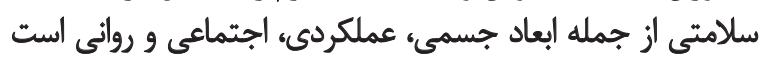

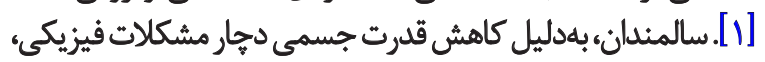

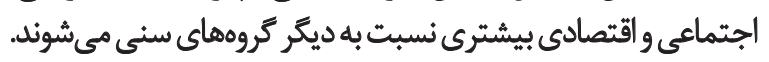

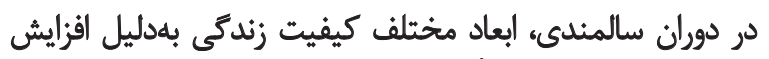

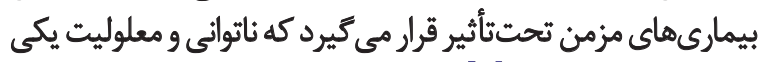

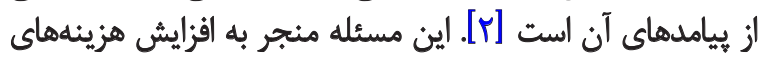

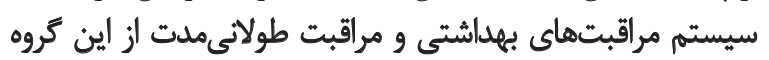

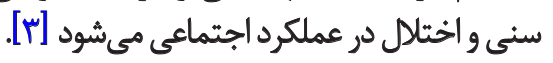

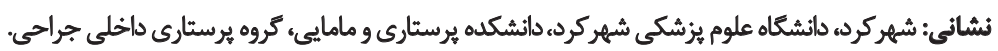

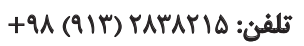
يست الكترونيكي: Ali20121968@yahoo.com 


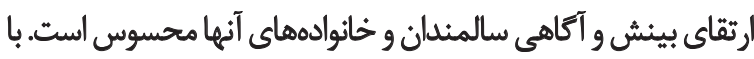

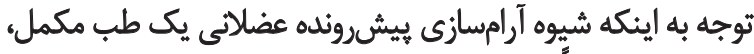

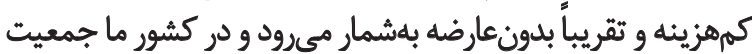

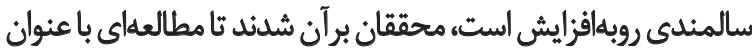

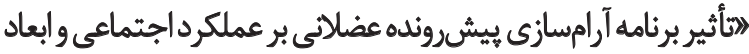
مختلف كيفيت زندكى سالمندان هانجام دهند.

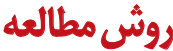

مطالعه نيمهتجربى حاضر با كسب مجوز لازم از معاونت تحقيقات

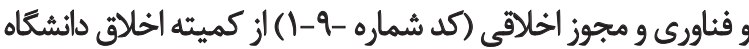

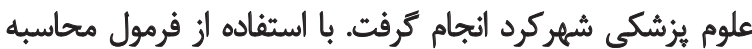

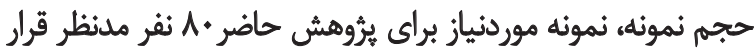

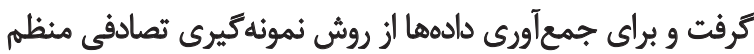

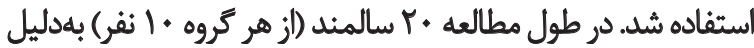

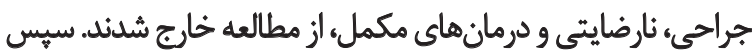

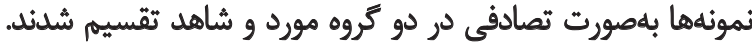

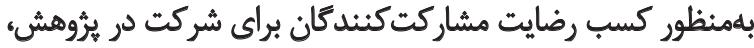

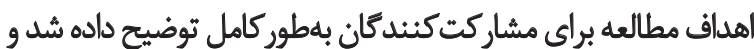

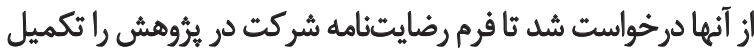

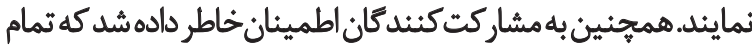

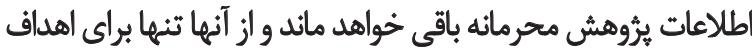
يرؤشش استفاده خواهد شد.

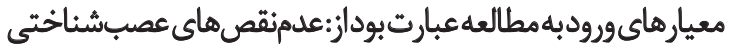

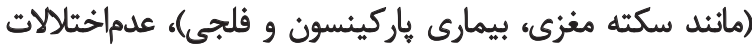

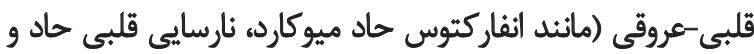

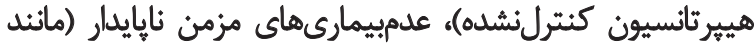

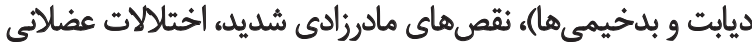

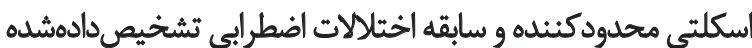

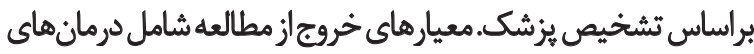

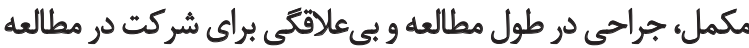

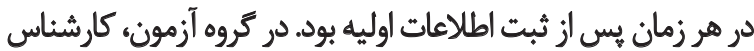

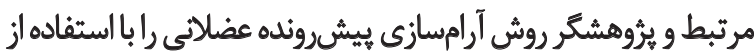

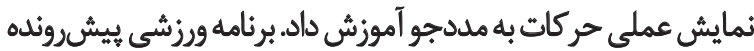

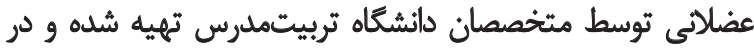

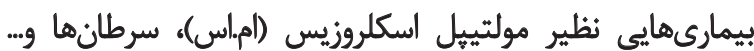

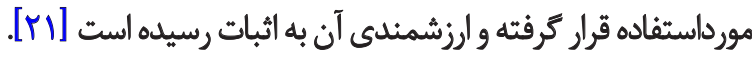

يس از اطمينان از درك و انجام صحيح روش، كاست صوتى و

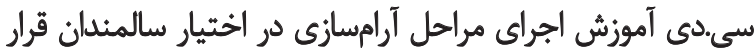

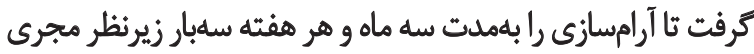

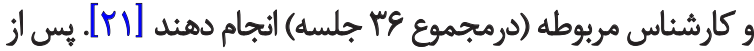

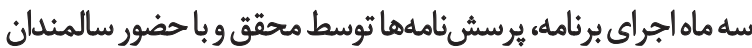

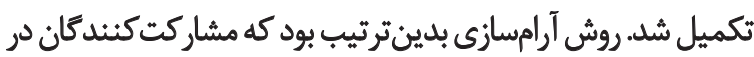

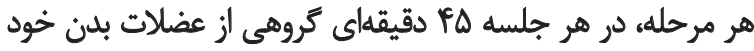

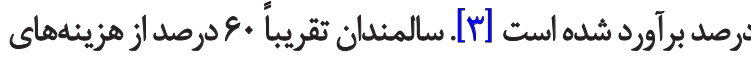

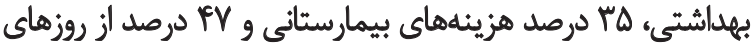

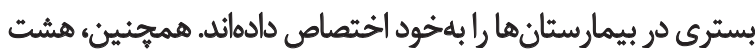

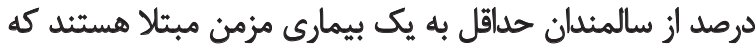

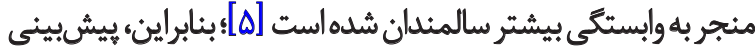

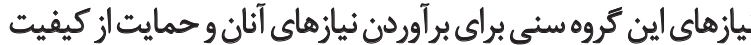

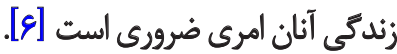

امروزه مفهوم جديدى از سلامت با عنوان بهبود كيفيت زندكى'

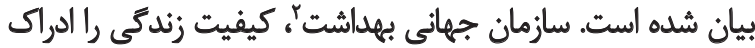

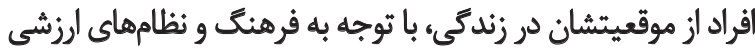

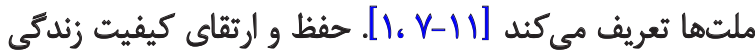

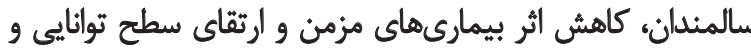

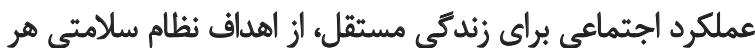

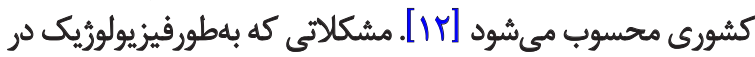

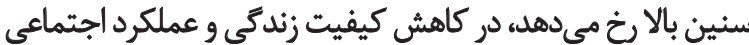

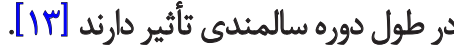
در روند درمان و مراقبت از سالمندان بايد به عوامل مؤثر در

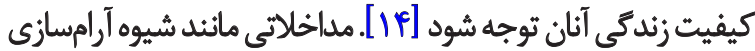

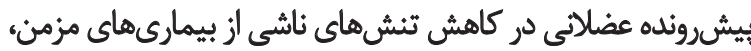

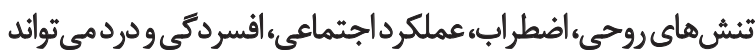

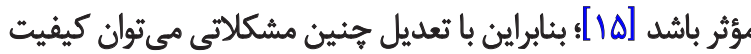

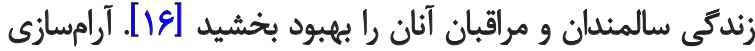

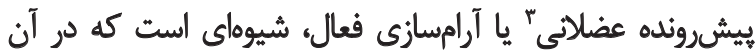

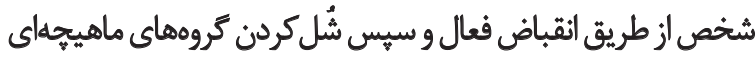

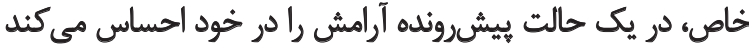

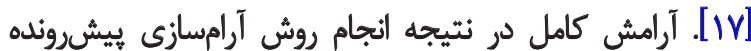

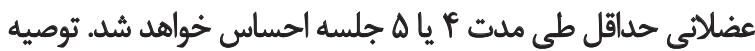

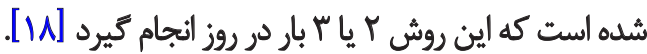
آرامسازى يكى از روشهاى طب مكمل و جايكزين است كه

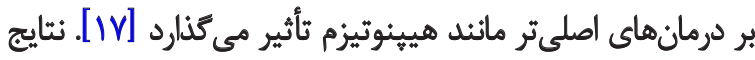

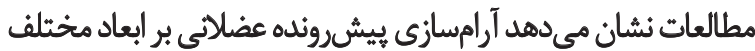

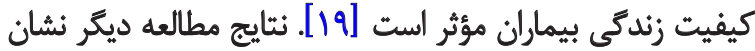

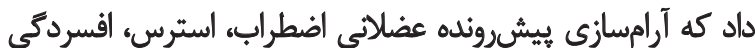

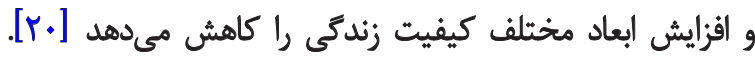

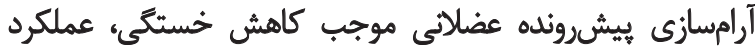

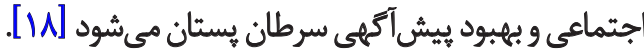

با وجود بيشرفتهاى جشمخيرى كه امروزه در زمينه كيفيت

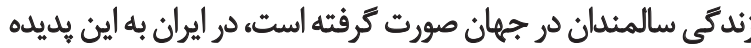
بلصورت سنتى توجه ميشودو كمبودمنابع علمى وحمايتى بلمنظوري

\section{Quality of life}

2. World Health Organization (WHO)

3. Progressive Muscle Relaxation (PMR) 
نتايج مطالعه نشان داد بين كروه مورد و شاهد قبل از مطالعها،

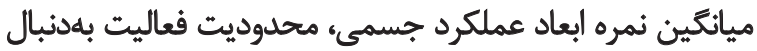

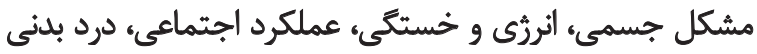

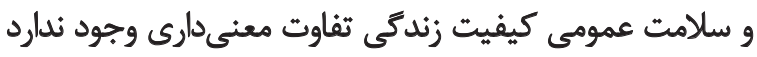

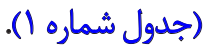

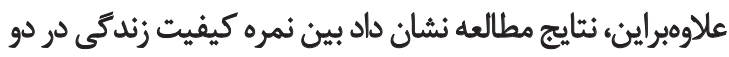

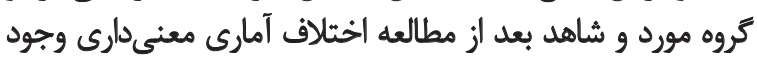

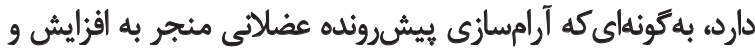

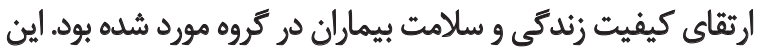

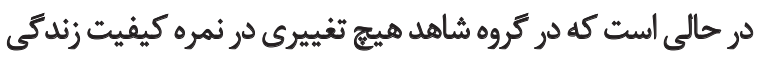

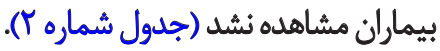

\section{بحث}

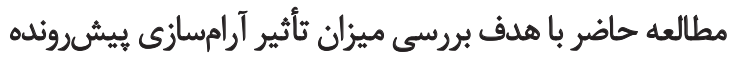

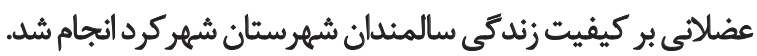

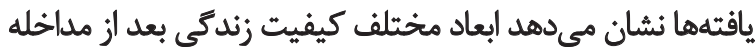

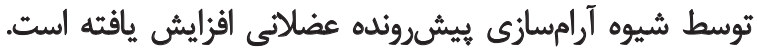

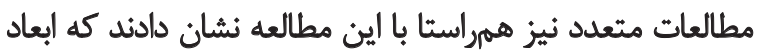

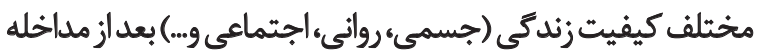
باشيوه مذكور، در بيماران مبتلا به مولتيخيل اسكلروزيس (امئاس)،

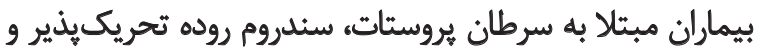

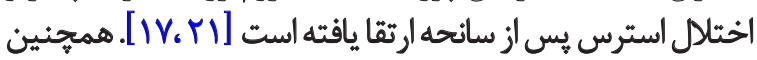

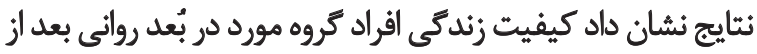
مداخله، بلهورفزايندهاي ارثقاي يافته است.

مطالعات محققان ديكر نيز نشان مى دهد كه شيوه آرامسازى

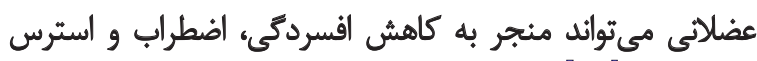

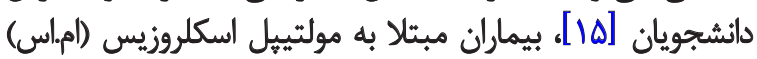

را منقبض و يس از ب ثا ها ثانيه رها و در باياين، تمام عضلات را شُل مى كردند و ينج بار نفس عميق مى آنشيدند.

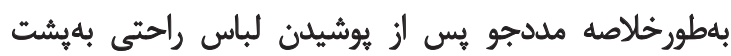

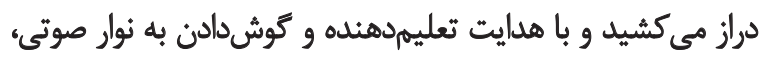

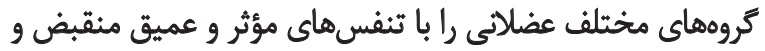

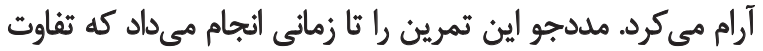

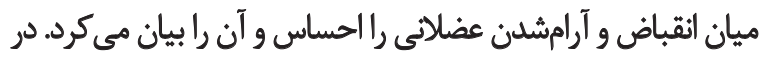

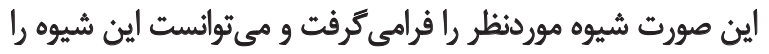

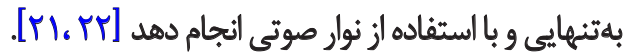

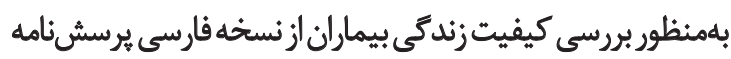

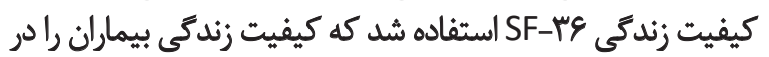

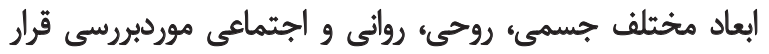

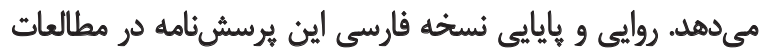

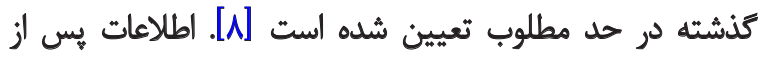

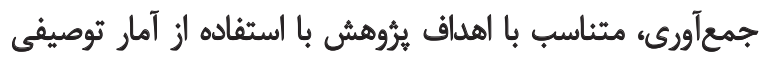

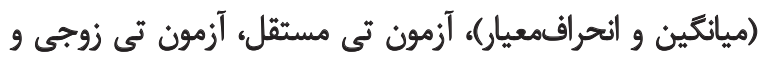

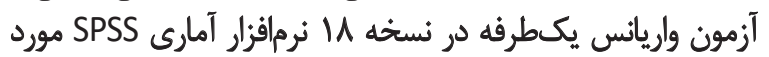

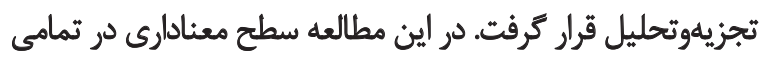

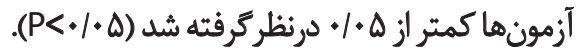

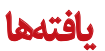

نثنايج مطالعه حاضر بيانكر آن بود كه ميانكين سنى كروه مورد

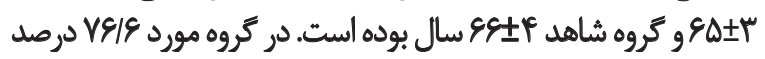

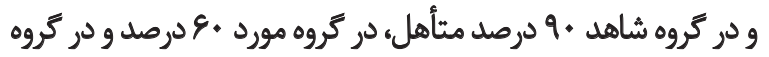

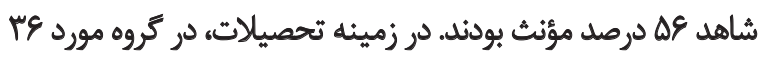

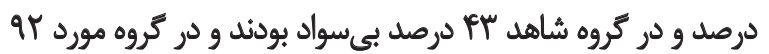
درصد ودر كروه شاهد الو درصد بر بيمه درمانى داشتند.

جدول ا. ميانكين و انحراف معيار نمره ابعاد مختلف كيفيت زندكى در دو كروه مورد و شاهد در بلو ورود به مطالعه.

\begin{tabular}{|c|c|c|c|c|}
\hline \multirow[b]{2}{*}{ نتيجه آزمون } & مئروه مورد & مئروه شاهد & \multirow[t]{2}{*}{ 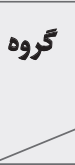 } & \\
\hline & ميانكين و انحراف معيار نمره & مياتكين و انفراق معيار نمره ابعاد & & \\
\hline$P>\cdot 10$ & $\operatorname{sep} 19 \pm v / p$ & 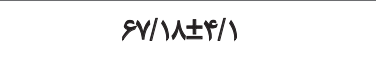 & & عملكردفيزيكى \\
\hline$P>\cdot 10$ & $f \Delta / q^{e} \pm V / f^{\circ}$ & $R T / M I \pm I / F$ & & ايفاى نقش \\
\hline $\mathrm{P}>\cdot 10$ & $8 / r+ \pm r / \Delta$ & $8 / / 19 \pm f / 0$ & & درد \\
\hline$P>\cdot 10$ & $\Delta \Psi / \backslash \Delta \pm T / r$ & $\Delta r / I P \pm V / r$ & & سلامت عمومى \\
\hline$P>\cdot 10$ & $F q / M \pm 1 / r$ & $P V / \Delta \pm \Delta / F$ & & سرزندكى و شادابي \\
\hline$P>\cdot 10$ & 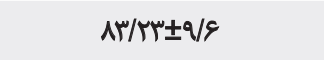 & $A r / T+ \pm F / V$ & & عملكرداجتماعى \\
\hline$P>+10$ & $r q \pm r \cdot 10$ & rq/TV $\pm V / T$ & & عملكرد عاطفى \\
\hline$P>\cdot 10$ & $M / / E \pm r / r$ & $r V / \backslash \notin \pm Y / \Psi$ & & سلامت روانى \\
\hline
\end{tabular}

L 
جدول r. ميانكين و انحراف معيار نمره ابعاد مختلف كيفيت زندكى در دو كروه مورد و شاهد در بعد از مطالعه.

\begin{tabular}{|c|c|c|c|c|}
\hline \multirow[b]{2}{*}{ تتيجه آزمون } & كروه مورد & كروه شاهد & \multirow[t]{2}{*}{ كروه } & \\
\hline & ميانكين و انحراف معيار نمره ابعادكيفيت زئدى & ميانكين و انحراف معيار نمره ابعاد كيفيت & & \\
\hline$P<+/ Q$ & $A r / I P \pm Y / Y$ & $8 / / V \pm \Psi / r$ & & عملكرد فيزيكى \\
\hline$P<\cdot / Q$ & EV/T $\Delta \pm \Delta / r$ & $R r / \Delta \pm Y \cdot / r$ & & ايفاى نقش \\
\hline$P<+/ Q$ & Whir \pm ep & $V / / V \pm r / \Delta$ & & درد \\
\hline$P<\cdot / \Delta$ & WNII $\pm \mathbb{M} / 1$ & $\Delta \backslash / / Y \pm=/ /$ & & سلامت عمومى \\
\hline$P<\cdot / Q$ & $r / l \cdot \pm r / r$ & $\Delta \Gamma / \backslash \Delta \pm \Psi / /$ & & سرزندكى و شادابي \\
\hline $\mathrm{P}<\cdot / \mathrm{Q}$ & $9 \vee / \Lambda \pm \Delta / \Delta$ & $A r / M \pm T / q$ & & عملكرد اجتماعى \\
\hline $\mathrm{P}<+/ \Delta$ & $V / R+ \pm N I$ & WNTA $\pm N$ & & عملكرد عاطفى \\
\hline$P<\cdot / \Delta$ & $g r / I r \pm 1 / r$ & $\mathbb{W} / \mathrm{If} \pm \mathrm{I} / \mathrm{V}$ & & 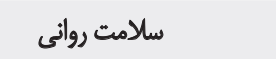 \\
\hline
\end{tabular}

(l)

به فرايند بيرى موفق و سالم آنان كمك كرد. ازاينرو، اجراى جنين

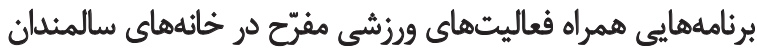

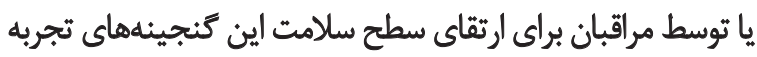

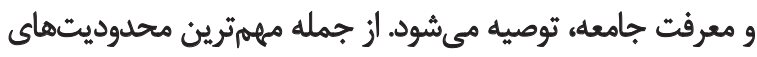
يرزوهش حاضر مى توان به تعداد كم نمونهها اشاره كرد كك تعميم نتايج

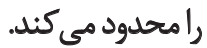

$$
\text { تشكرو ثدردانئ. }
$$

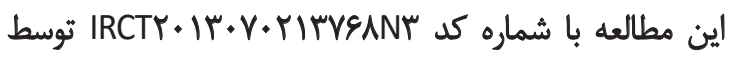

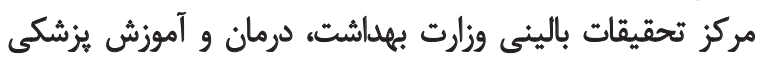

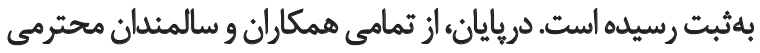

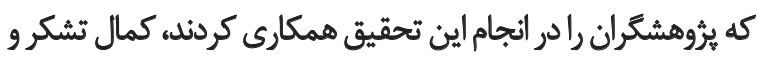

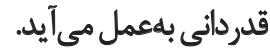

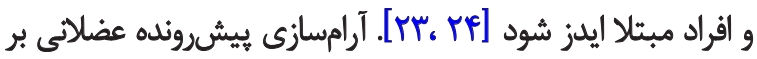

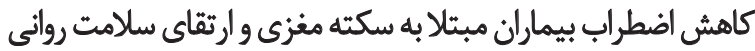

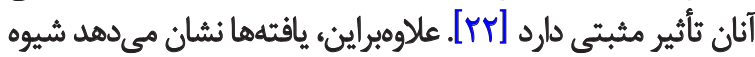

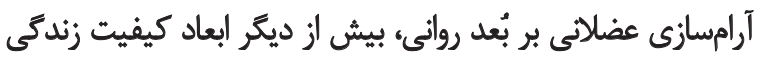

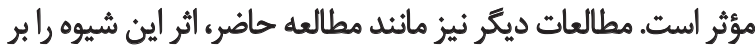

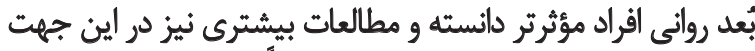

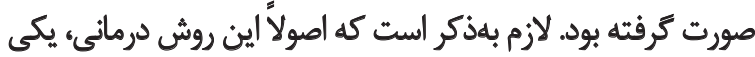

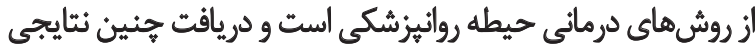

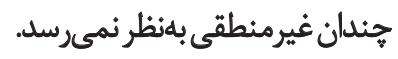

اين مطالعه، آرامسازى عضلانى راروشى برائى كاهش دردي درد سالمندان

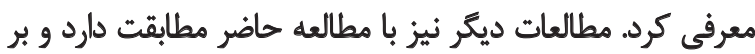

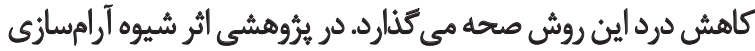

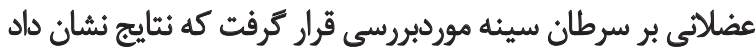

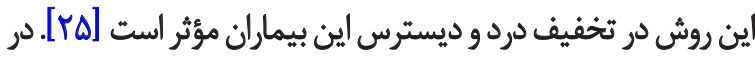

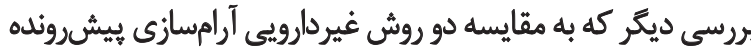

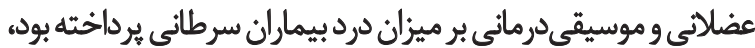

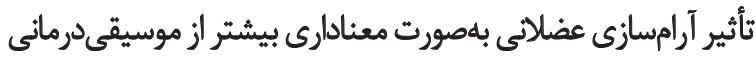

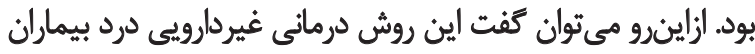

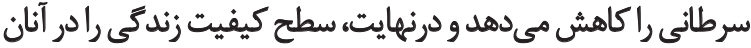

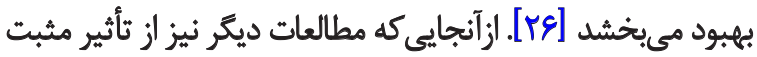

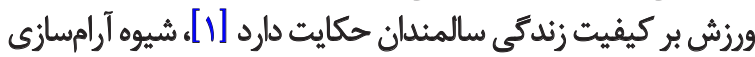

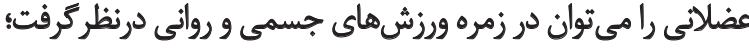
بنابراين، انجام مرتب آن در قشر سالمند توصيه مي جئود.

\section{نتيجهلَّيرىنهايي}

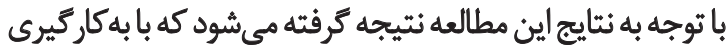

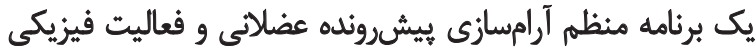

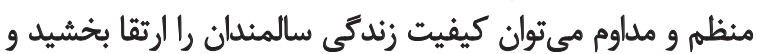

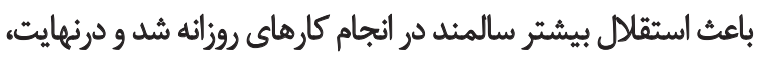




\section{References}

[1] Heydarnijad S, Dehkordi AH. The effect of an exercise program on the health-quality of life in older adults: a randomized controlled trial. Danish Medical Bulletin. 2010; 57(3):4113-117.

[2] Langlois F, Vu TM, Chassé K, Dupuis G, Kergoat MJ, Bherer L. Benefits of physical exercise training on cognition and quality of life in frail older adults. Journals of Gerontology Series B: Psychological Sciences \& Social Sciences. 2013; 68(3):400-04.

[3] Noroozian M. The elderly population in Iran: an ever growing concern in the health system. Iranian Journal of Psychiatry and Behavioral Sciences. 2012; 6(2):11-23.

[4] Statistical Center of Iran. [Selected results of national population and housing census in 2012 Tehran (Persian)] [Internet]. 2012 [cited 2016 Sep. 25]. Available from: http:/ / iran.unfpa.org/Documents/Census2011/census-90-results(3).pdf

[5] Ahmadi F, Salar A, Faghihzadeh S. [Quality of life in Zahedan elderly population (Persian)]. Hayat. 2004; 10(3):61-67.

[6] Tajvar M, Arab M, Montazeri A. Determinants of health-related quality of life in elderly in Tehran, Iran. BMC Public Health. 2008 8:323. doi: 10.1186/1471-2458-8-323

[7] Dehkordi AH, Heydarnejad MS, Fatehi D. Quality of life in cancer patients undergoing chemotherapy. Oman Medical Journal. 2009; 24(3):204-11.

[8] Dehkordi AH, Far AK. Effect of exercise training on the quality of life and echocardiography parameter of systolic function in patients with chronic heart failure: a randomized trial. Asian Journal of Sports Medicine. 2015; 6(1):43-56.

[9] Dehkordi AH, Heydarnejad MS. Factors-related to quality of life in post myocardial infarction patients. Journal of Hainan Medical College. 2009; 7(3):10-14

[10] Dehkordi A, Jivad N. Comparison of regular aerobic and yoga on the quality of life in patients with multiple sclerosis. Medical Journal of Islamic Republic of Iran. 2014; 28(1):141-47.

[11] Heydarnejad M, Hassanpour DA, Solati DK. Factors affecting quality of life in cancer patients undergoing chemotherapy. African Health Sciences. 2012; 11(2):266-70.

[12] Burack OR, Weiner AS, Reinhardt JP, Annunziato RA. What matters most to nursing home elders: quality of life in the nursing home. Journal of the American Medical Directors Association. 2012; 13(1):48-53.

[13] Landi F, Liperoti R, Fusco D, Mastropaolo S, Quattrociocchi D, Proia A, et al. Prevalence and risk factors of sarcopenia among nursing home older residents. Journals of Gerontology Series A: Biological Sciences \& Medical Sciences. 2012; 67(1):48-55.

[14] Ciorba A, Bianchini C, Pelucchi S, Pastore A. The impact of hearing loss on the quality of life of elderly adults. Clinical Interventions in Aging. 2012; 7(3):159-63.

[15] Dehkordi AH, Heydarnejad S. Effect of relaxation on the level of nursing internship students. Journal of Hainan Medical College. 2010; 16(1):20-22.

[16] Kempen GI, Ballemans J, Ranchor AV, van Rens GH, Zijlstra GR. The impact of low vision on activities of daily living, symptoms of depression, feelings of anxiety and social support in community-living older adults seeking vision rehabilitation services. Quality of Life Research. 2012; 21(8):1405-411.
[17] Dayapoğlu N, Tan M. Evaluation of the effect of progressive relaxation exercises on fatigue and sleep quality in patients with multiple sclerosis. Journal of Alternative \& Complementary Medicine. 2012; 18(10):983-87.

[18] Potthoff K, Schmidt ME, Wiskemann J, Hof H, Klassen O, Habermann N, et al. Randomized controlled trial to evaluate the effects of progressive resistance training compared to progressive muscle relaxation in breast cancer patients undergoing adjuvant radiotherapy: the BEST study. BMC Cancer. 2013; 13(1):162-69.

[19] Mazloomy Mahmoodabad S, Zolghadr R, Mirhossaini J, Yasini Ardakani S, Fallahzadeh H, Askarifar J. [Effect of Benson relaxation education on quality of life in patients after open heart surgery in Yazd city (Persian)]. Tolooe Behdasht. 2014; 12(4):88-96.

[20] Zhao L, Wu H, Zhou X, Wang Q, Zhu W, Chen J. Effects of progressive muscular relaxation training on anxiety, depression and quality of life of endometriosis patients under gonadotrophin-releasing hormone agonist therapy. European Journal of Obstetrics \& Gynecology \& Reproductive Biology. 2012; 162(2):211-15.

[21] Isa MR, Moy FM, Abdul Razack A, Zainuddin ZM, Zainal NZ. Impact of applied progressive deep muscle relaxation training on the level of depression, anxiety and stress among prostate cancer patients: a quasi-experimental study. Asian Pacific Journal of Cancer Prevention. 2013; 14(4):2237-242.

[22] Jariani M, Saki M, Momeni N, Ebrahimzade F, Seydian A. [The effect of progressive muscle relaxation techniques on anxiety in patients with myocardial infarction (Persian)]. Yafteh. 2011; 13(3):22-30

[23] Bommareddi P, Valsaraj B. Progressive muscle relaxation (JPMR) training to reduce anxiety and depression among people living with HIV. Nitte University Journal of Health Science. 2014; 4(1):72-78.

[24] Ghafari S, Ahmadi F, Nabavi M, Memarian R. [Effects of applying progressive muscle relaxation technique on depression, anxiety and stress of multiple sclerosis patients in Iran National MS Society (Persian)]. Research in Medicine. 2008; 32(1):45-53.

[25] Hajian S, Mirzaii N, Keramat A, Mirzaii H. [The effect of muscle relaxation and guided imagery on pain and distress reduction caused by disease and treatment side effects in women with breast cancer during 1998-2007: a systematic review (Persian)]. Iranian Journal of Breast Disease. 2008; 1(3):32-44.

[26] Shaban M, Rasoolzadeh N, Mehran A, Moradalizadeh F. [Study of two non-pharmacological methods, progressive muscle relaxation and music, on pain relief of cancerous patients (Persian)]. Hayat. 2006; 12(3):63-72. 